\title{
Non-targeted Parallel Cascade Selection Molecular Dynamics based on a Non- Redundant Selection Rule for Initial Structures Enhances Conformational Sampling of Proteins
}

\author{
Ryuhei Harada ${ }^{*} \dagger$, Vladimir Sladek ${ }^{*} \$$, and Yasuteru Shigeta ${ }^{*}, \dagger$ \\ ${ }^{\dagger}$ Center for Computational Sciences, University of Tsukuba \\ 1-1-1 Tennodai, Tsukuba, Ibaraki 305-8577, Japan \\ §Institute of Chemistry - Centre for Glycomics, Dubravska cesta 9, 84538 Bratislava, \\ Slovakia \\ ${ }^{\sharp}$ Agency for Medical Research and Development (AMED), 1-7-1 Chiyoda-ku, Japan \\ Corresponding authors: Ryuhei Harada, Vladimir Sladek, and Yasuteru Shigeta \\ E-mail: ryuhei@ccs.tsukuba.ac.jp, sladek.vladimir@savba.sk, shigeta@ccs.tsukuba.ac.jp
}


Free Energy Calculation based on a Combination of the Extended/Original nt-PaCs-MD and Markov State Model

Generally, the extended/original nt-PaCS-MD cannot estimate free energy landscapes (FELs) directly because the trajectories generated by short-time MD simulations are independent and discontinuous. After the conformational search by the extended/original nt-PaCS-MD, the sampled protein configurations should be quantitatively evaluated on a FEL as a function of a set of reasonable reaction coordinates (RCs). A viable strategy to calculate the FEL is to construct a reliable Markov state model (MSM). The construction of the MSM consists of the following two steps, i.e. (1) defining microstates and (2) choosing a reasonable lag time $(\tau)$. First, the protein configurations generated by nt-PCS-MD are clustered by the $k$-means algorithm to yield $N$ clusters (the definition of $N$ microstates), and then each protein configuration is assigned to the closest microstates (clusters). To determine the appropriate lag time $\tau$, the $i^{\text {th }}$ slowest implied time, $t_{i}$, estimated from the $i^{\text {th }}$ largest eigenvalue, $\lambda_{i}(\tau)$, of the $N \times N$ transition matrix $\boldsymbol{T}$ defined as

$$
t_{i}(\tau)=\frac{-\tau}{\ln \lambda_{i}(\tau)}
$$

is monitored by changing $\tau$ until $t_{i}(\tau)$ reaches an approximately constant value, meaning that a given system satisfies the Markov assumption. By specifying the reasonable $\tau$, transitions among the microstates are again counted to estimate the maximum likelihood transition matrix $\boldsymbol{T}$ under the constrained detailed balance.

To estimate the FEL, a normalized equilibrium distribution $\pi=\left\{\pi_{i}\right\}\left(\sum_{i} \pi_{i}=1\right)$ are estimated from $\boldsymbol{T}$, which fulfils $\boldsymbol{\pi}=\boldsymbol{T} \boldsymbol{\pi}$, meaning that $\boldsymbol{\pi}$ is obtained from one of the eigenvectors of $\boldsymbol{T}$. Finally, the FEL is calculated as follows:

$$
F_{i}=-k_{B} T \ln \frac{\pi_{i}}{\max _{j} \pi_{j}}(i=1,2, \ldots, N)
$$

where the origin of FEL is selected as the maximum value of $\pi$ in the present study.

To evaluate the protein configurations generated by the extended/original nt-PaCS-MD based 
on mRMSD, a set of two $\mathrm{C}_{\alpha}$ RMSDs measured from the open/closed structures of $\mathrm{T} 4$ lysozyme (RMSD $_{\text {open, }} \mathrm{RMSD}_{\text {closed }}$ ) was specified as RCs and FELs were calculated as a function of these RCs. To construct an MSM, the MSM builder EMMA ${ }^{1}$ was utilized, where the resulting MSM contains 50 microstates obtained by the $k$-means clustering algorithm, i.e., $N=50$. To count structural transitions among the microstates, we considered all the trajectories generated by the five the extended/original nt-PaCS-MD runs based on mRMSD $\left(N_{\text {initial }}=20\right.$ and 25 cycles $)$. To construct reliable MSMs, we calculated the implied times defined by Eq. (1) as a function of $\tau$. As shown in Figs. S1(a-b), the slowest top two implied timescales, $\left\{t_{i}(\tau)\right\}(i=1,2)$ in Eq. (1), reached almost constant values after $25 \mathrm{ps}$ in both cases. Therefore, we set a reasonable $\tau$ to $25 \mathrm{ps}$ to construct the reliable MSMs using the trajectories generated by the extended/original nt-PaCS-MD.

Based on the constructed MSMs, FELs for characterizing the open-closed transition of T4 lysozyme were estimated on the two-dimensional subspace by obtaining equilibrium distributions, i.e., $\pi=\left\{\pi_{i}\right\}(i=1,2, \ldots, N=50)($ see Figs. $6(\mathrm{a}-\mathrm{b})$ in the main text). 
Figure S1
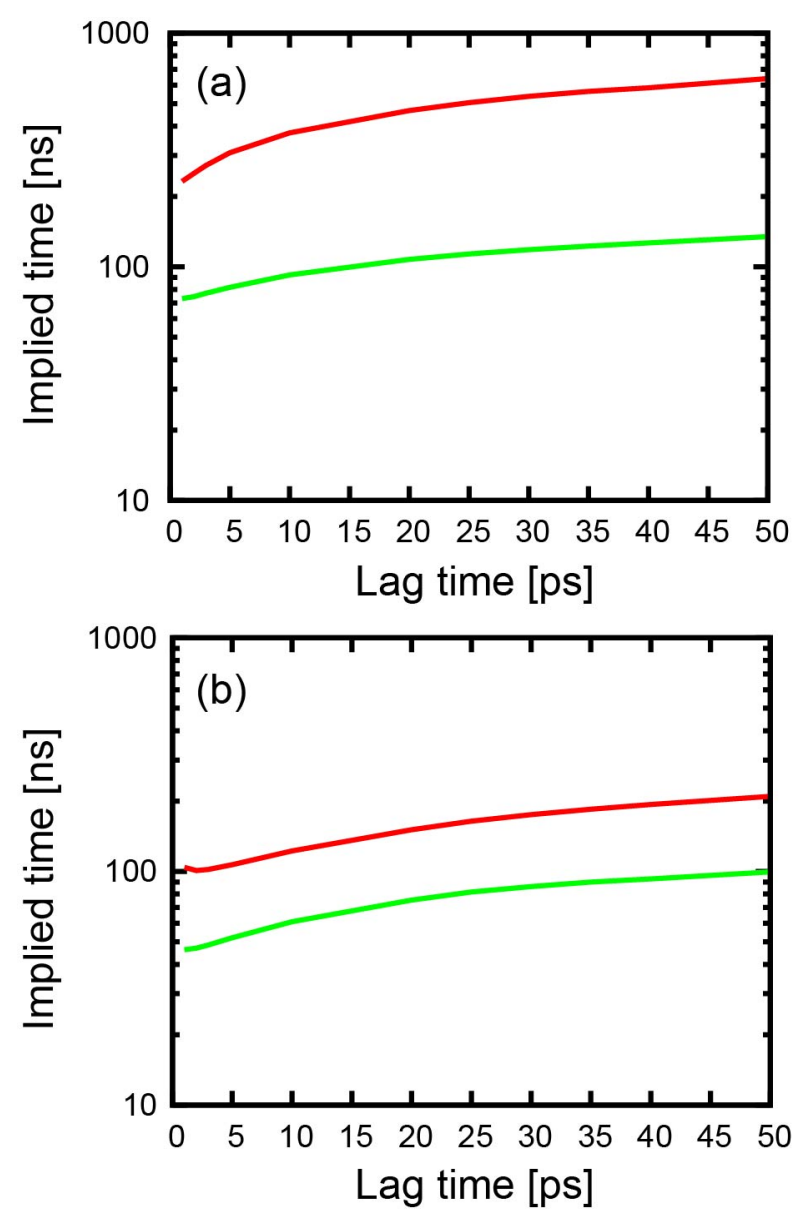

Profiles of the slowest top two implied times as a function of lag time, $\left\{t_{i}(\tau)\right\}(i=1,2)$ in Eq. (1). (a) The extended nt-PaCS-MD. (b) The original nt-PaCS-MD. 


\section{Reference}

1. Senne, M.; Trendelkamp-Schroer, B.; Mey, A. S. J. S.; Schutte, C.; Noe, F. Emma: A Software Package for Markov Model Building and Analysis. J. Chem. Theory Comput. 2012, 8, 22232238. 